\title{
Lymphatic function measurements influenced by contrast agent volume and body position
}

Echoe M. Bouta, ${ }^{1,2,3}$ Cedric Blatter, ${ }^{3,4}$ Thomas A. Ruggieri, ${ }^{2}$ Eelco F.J. Meijer, ${ }^{1,2,3}$ Lance L. Munn, ${ }^{1,2,3}$ Benjamin J. Vakoc, ${ }^{3,4}$ and Timothy P. Padera ${ }^{1,2,3}$

${ }^{1}$ Edwin L. Steele Laboratories, and ${ }^{2}$ Department of Radiation Oncology, MCH Cancer Center, Massachusetts Ceneral Hospital, Boston, Massachusetts, USA. ${ }^{3}$ Harvard Medical School, Boston, Massachusetts, USA. ${ }^{4}$ Wellman Center for Photomedicine, Massachusetts General Hospital, Boston, Massachusetts, USA.

Several imaging modalities have been used to assess lymphatic function, including fluorescence microscopy, near-infrared fluorescence (NIRF) imaging, and Doppler optical coherence tomography (DOCT). They vary in how the mouse is positioned, the invasiveness of the experimental setup, and the volume of contrast agent injected. Here, we present how each of these experimental parameters affects functional measurements of collecting lymphatic vessels. First, fluorescence microscopy showed that supine mice have a statistically lower contraction frequency compared with mice sitting upright. To assess the effect of different injection volumes on these endpoints, mice were injected with 4,10 , or $20 \mu$ of dye. The lowest frequencies were observed after 20 - $\mu$ l injections. Interestingly, lymph-flow DOCT revealed that although there was lower contraction frequency in mice injected with $20 \mu \mathrm{l}$ versus $4 \mu \mathrm{l}$, mice showed a higher volumetric flow with a 20 - $\mu$ l injection. This indicates that contraction frequency alone is not sufficient to understand lymphatic transport. Finally, NIRF revealed that removing the skin reduced contraction frequency. Therefore, this study reveals how sensitive these techniques are to mouse position, removal of skin, and dye volume. Care should be taken when comparing results obtained under different experimental conditions.

\section{Introduction}

The lymphatic system has become heavily researched due to its potential role in many disease processes, including Alzheimer's disease, rheumatoid arthritis, glaucoma, hypertension, myocardial infarction, lymphedema, and cancer (1-6). This research focus has catalyzed the advancement of techniques used to assess lymphatic function (7), including near-infrared fluorescence (NIRF) imaging, direct fluorescence imaging of lymphatic contraction, and Doppler optical coherence tomography (DOCT) (8-10). NIRF imaging is used both preclinically and clinically to visualize how injected NIR contrast agents — frequently the FDA-approved molecule indocyanine green (ICG) - move though lymphatic vessels (11-13). A region of interest (ROI) on the vessel is used to measure the changes in intensity that likely correspond to lymphatic contraction and/or movement of lymph fluid (14). An advantage of NIRF imaging is that it allows for imaging noninvasively through the skin. However, due to photon scattering in the skin (15), it is largely used for macroscopic analysis (i.e., entire mouse limb or large sections of a patient arm).

For microscopic analysis, fluorescence imaging of lymphatic contraction is possible after removing the overlying skin; high-resolution $(\times 20)$ images can then be acquired of a single vessel over time. This allows for the

Conflict of interest: The authors have declared that no conflict of interest exists.

Submitted: July 31, 2017

Accepted: December 14, 2017

Published: January 25, 2018

Reference information:

JCI Insight. 2018;3(2):e96591. https:// doi.org/10.1172/jii.insight.96591. assessment of wall movement, permitting quantification of contraction frequency and ejection fraction (EF; percentage of volume change per contraction) $(9,16)$. DOCT is unique in that, contrary to the NIRF and fluorescence imaging of lymphatic contraction, no dye is needed to measure the output of volumetric flow (17). There are many differences between experimental parameters used for these techniques, including mouse position, volume of dye injected, invasiveness of the preparation, and data analysis (Table 1).

While these advancements in imaging have helped define lymphatic physiology in normal and disease states, there is wide variability in the published data when comparing between these techniques, even among common outputs such as contraction frequency. This may be due to the lack of standardized protocols or inability to control for critical experimental variables in vivo. Studies have shown that lymphatics respond to a wide 
Table 1. Comparison of lymphatic function measurement techniques

\begin{tabular}{cccc}
\hline Technique & $\begin{array}{c}\text { Lymphatic contraction } \\
\text { uses wall motion to measure } \\
\text { Analysis }\end{array}$ & frequency measured by ROI intensity & $\begin{array}{c}\text { NoCT } \\
\text { analysis of Doppler frequency } \\
\text { shifts of back-scattered light }\end{array}$ \\
Mouse position & sitting upright & supine & prone at a $15^{\circ}$ angle \\
Invasiveness & skin removed & imaging through skin & skin removed \\
Dye & $2 \mu$ l FlTC-dextran & $2-10 \mu l$ ICC or other NIR dye & none needed \\
References & $(9,16)$ & $(11-14)$ & $(8-10)$
\end{tabular}

DOCT, Doppler optical coherence tomography; ICG, indocyanine green; NIRF, near-infrared fluorescence.

A

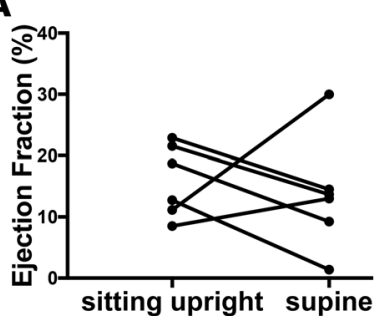

variety of stimuli - including pressure, flow, and stretch (18-21) - indicating that a variety of experimental parameters (Table 1) may be influential. Several studies have sought to characterize how some experimental parameters affect lymphatic functional measurements. These include studies that explored the effect of tracer type and size (13), ICG concentration (22), and whether the fluorophore, time after injection, and vessel selection alter the measurements (23). However, these studies did not compare different measurement techniques. Therefore, we sought to quantify how experimental parameters - including mouse position, skin removal, and volume of injected contrast agent — affect the results obtained with NIRF imaging, direct fluorescence imaging of lymphatic contraction, and DOCT, and how different methods are more or less sensitive to these parameters.

\section{Results}

First, we assessed how mouse position with respect to gravity affects measurements of lymphatic function. While most techniques are performed in the prone or supine position, fluorescence imaging can also measure lymphatic contraction with mice sitting upright $(9,16)$, with the afferent lymphatic to the popliteal lymph node gravitationally dependent. Therefore, we positioned mice either sitting upright or supine while they underwent fluorescence imaging of lymphatic contraction. It was found that the supine position lowers the frequency, although no effect was found in EF (Figure 1).

We then evaluated whether the volume of injected contrast affects measurements of lymphatic function. Mice were injected with 4, 10, or $20 \mu \mathrm{l}$ of a 50:50 solution of ICG and FITC-dextran, enabling both NIRF imaging (Figure 2) and fluorescence imaging of lymphatic contraction (Figure 3) in the same animal. NIRF imaging showed that the lowest contraction frequency was measured after an injection of $20 \mu 1$ of contrast agent (Figure 2D). This is similar to what others have reported in ex vivo measurements of lymphatic vessels when a favorable pressure gradient was imposed, likely causing a shear-dependent response (18, 20, 24 26). (Figure 2, A-D and Supplemental Videos 1-3; supplemental material available online with this article;

\section{B}

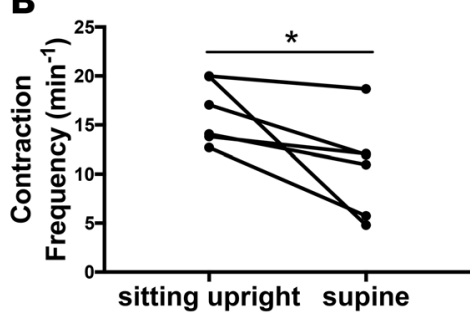

Figure 1. Mice in the supine position demonstrated lower lymphatic contraction frequency compared with mice sitting upright by fluorescence imaging of lymphatic contraction. Mice were injected with $3 \mu \mathrm{l}$ of FITC-dextran and underwent lymphatic contraction analysis both while sitting upright and in the supine position. The order of the positioning was randomized. Subsequently, analysis of ejection fraction (A) and contraction frequency (B) was performed. Notice that the contraction frequency is lower when mice are in the supine position versus sitting upright (B, ${ }^{*} P<0.05$ by a paired $t$ test, $n=6$ ). https://doi.org/10.1172/jci.insight.96591DS1). More vessels are visible in the NIRF imaging area when larger volumes of dye are injected (Figure 2C vs. Figure 2A). We then assessed if removing skin and superfusing the vessels with sterile saline affected lymphatic contraction. By removing the skin, contraction frequencies measured by NIRF were reduced (Figure 2D). This is likely due to the superfusion of vessels with saline or from a reduction in confinement/extramural compression by removing the skin.

Mice then underwent direct measurement of lymphatic contraction by fluorescence microscopy. It was found that increasing the volume of injected contrast agent increased lymphatic vessel diameter, but that difference was only detectable when the mice were sitting upright (Figure 3, A-D and Supplemental Videos 4-6). Similar results of increased lymphatic diameter following edemagenic stress have been reported (27). Further, the lowest contraction frequency was measured after an injection of $20 \mu \mathrm{l}$ of contrast agent when the mice were positioned sitting upright (Figure $3 \mathrm{~F}$ ), similar to the results 

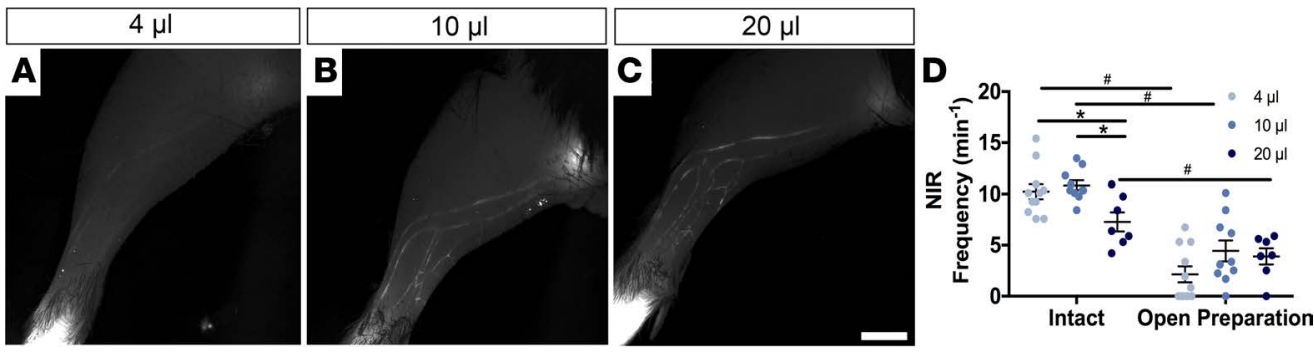

Figure 2. Injection volume and skin removal affects frequency by NIRF imaging. Mice injected with a 50:50 mixture of FITC-dextran and indocyanine green (ICG) underwent near-infrared fluorescence (NIRF) imaging with subsequent fluorescence contraction imaging while sitting upright or supine. (A-C) Intact representative NIRF images are shown after mice were injected with (A) $4 \mu \mathrm{l}$, (B) $10 \mu \mathrm{l}$, or (C) $20 \mu \mathrm{l}$ (Supplemental Videos 1-3, respectively). (D) NIR frequency is affected by volume injected. In general, removing the skin and superfusing with saline decreases the contraction frequency. Data presented as the mean \pm SEM. ${ }^{*} P<0.05$ by 2 -way ANOVA with a Tukey's multiple comparison test; ${ }^{\#} P<0.05$ by a paired $t$ test; $n=7-11$. Scale bar: 5 mm.

with NIRF imaging (Figure 2D). However, mice in the supine position did not show a decrease in contraction frequency when they underwent direct measurement of lymphatic contraction (Figure 3F). As mice in the supine position show lower frequency via direct measurement of lymphatic contraction (Figure 1), it is possible that the lower frequency masks any further decrease in frequency that may occur in response to greater injection volumes. Interestingly, a weak correlation was found between NIR with intact skin and fluorescence intravital contraction frequency measurements with the mouse sitting upright (Figure 4).

We then assessed whether there were any differences in volumetric flow for the different injection volumes when measured using DOCT. The mean volumetric flow was lower in mice injected with $4 \mu \mathrm{l}$ compared with $20 \mu \mathrm{l}$, with the minimum flow (Figure 5, A and C, respectively) and peak flow (Figure 5, B and D, respectively) both showing this difference. Minimum flow occurs between contractions, while peak flow occurs immediately following a contraction (17). Volumetric flow rate is quantified from DOCT measurements by using the instantaneous cross-sectional vessel area and instantaneous velocity averaged over the vessel cross section (Figure 5, E and F and Supplemental Videos 7 and 8 , respectively). The mean volumetric flow averaged over the time of the imaging session was found to be higher in mice injected with $20 \mu \mathrm{l}$ compared with $4 \mu \mathrm{l}$ (Figure 5G). However, there was no difference in the frequency of peaks of volumetric flow between the 2 groups (Figure $5 \mathrm{H}$ ). These results are in contrast to the contraction analysis, which showed a lower contraction frequency in $20 \mu \mathrm{l}$

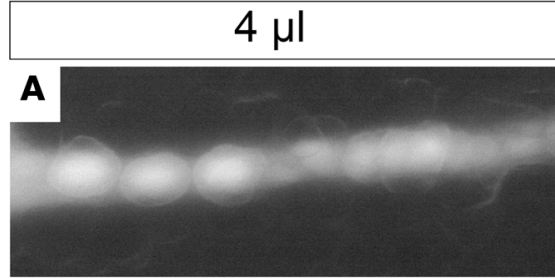

D

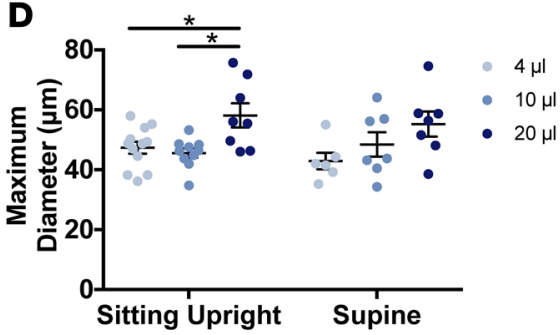

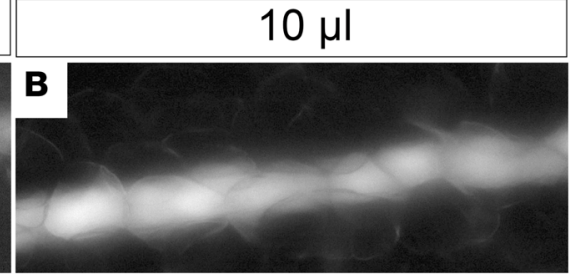

E

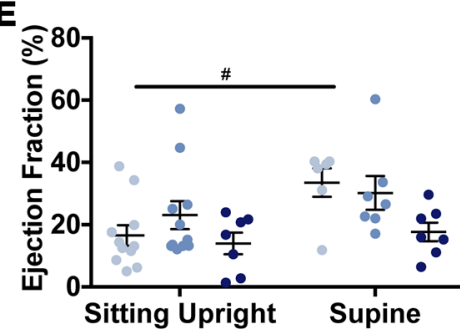

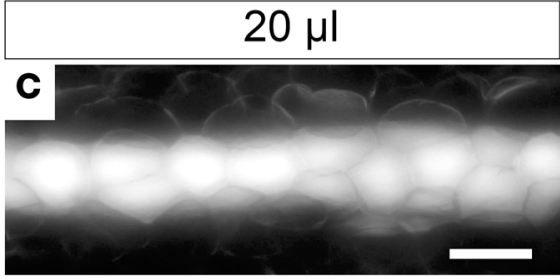

$\mathbf{F}$

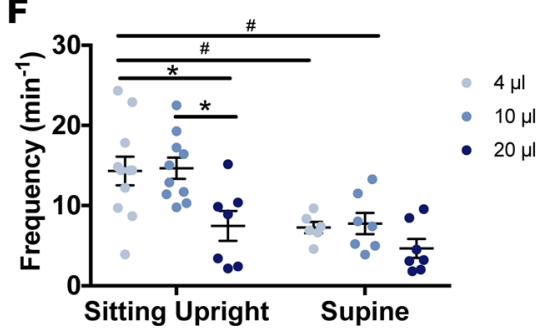

Figure 3. Injection volume affects contraction frequency. The same mice in Figure 2 underwent fluorescence contraction imaging following near-infrared fluorescence (NIRF) imaging. Representative FITC images of lymphatic vessels from mice injected with $4 \mu \mathrm{l}, 10 \mu \mathrm{l}$, or $20 \mu \mathrm{l}$ of dye are shown (A-C, respectively). (D) Mice injected with $20 \mu \mathrm{l}$ showed larger lymphatic vessel diameters. (E) Ejection fraction and (F) contraction frequency were quantified from intravital videos of lymphatic wall motion (Supplemental Videos 4-6). Mice injected with $20 \mu \mathrm{l}$ showed lower contraction frequency compared with mice injected with 4 and $10 \mu \mathrm{l}$. Data presented as the mean \pm SEM. ${ }^{*} P<0.05$ by ANOVA with a Fisher's LSD post-hoc test; ${ }^{*} P<0.05$ by Mann-Whitney test; $n=6-11$. Scale bar: $50 \mu$ m. 


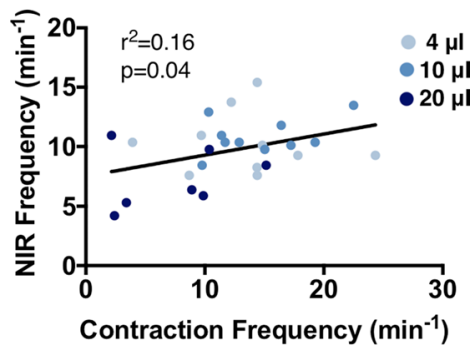

Figure 4. NIR and direct measurement of lymphatic contraction are correlated. Analysis was performed on mice from Figures 2 and 3. A weak correlation was found between near infrared (NIR) with intact skin and fluorescence intravital contraction frequency measurements with the mouse sitting upright ( $P$ value found by linear regression, $n=6-11$ ). These conditions are the ones most commonly found in the literature for each technique.

compared with $4 \mu \mathrm{l}$ (Figure 2D and Figure 3F). These data show that flow measurements are critical to fully describing lymphatic function and lymph transport, and further suggest that there is not a one-toone correlation between vessel contractions and peaks in flow.

\section{Discussion}

Here we show how experimental parameters can dramatically affect the endpoints of NIRF imaging, direct fluorescence imaging of lymphatic contraction, and DOCT. Mouse position was found to be important, as contraction frequency was higher when the mouse was sitting upright versus in the supine position (Figure 1B). This could be due to a higher pressure gradient imposed by gravity that the vessels have to contract against when sitting upright, recapitulating results from ex vivo preparations (19). Interestingly, increasing the volume of fluid injected in the footpad reduced the NIR frequency and contraction frequency (Figure $2 \mathrm{D}$ and Figure $3 \mathrm{~F}$ ). This is likely due to increases in pressure, stretch of the vessel and/or increased shear as greater volumes are injected $(25,28)$. It has previously been hypothesized that increasing tissue pressure increases lymphatic pressure (27). Importantly, this seminal work shows that the lymphatics rapidly respond to changes in pressure (27). Others have shown similar patterns in frequency, amplitude, and fractional pump flow with an increased driving pressure gradient using ex vivo preparations (18, 20, 24, 28). However, these results contradict ex vivo experiments that show an increase in frequency following increases in preload without changes in afterload, suggesting that increasing the volume injected does not simply increase preload $(19,29)$.

Importantly, similar patterns were seen across direct fluorescence contraction measurements and NIRF imaging following various volume injections (Figure 2D and Figure 3F), resulting in a significant correlation between the techniques when performed as previously published (i.e., NIRF with an intact preparation and direct measurement of lymphatic contraction with the skin removed and the mouse sitting upright) (Figure 4). However, this correlation was poor $\left(r^{2}=0.16, P=0.04\right)$, suggesting that while these techniques show the same general trends for a given experimental group, they are ultimately measuring different components of lymphatic functionality. It was previously reported that NIRF intensity changes in an ROI and wall motion were correlated (14). This correlation was made using the same videos of a contracting lymphatic, eliminating any differences in imaging technique, such as magnification, light source, or sensitivity of the system. In our comparison, we are imaging at different spatial resolutions and using different detectors, which may alter the overall sensitivity of a system. Based on the lower resolution of our NIR system, there is a differential sensitivity, which may be contributing to the lack of high correlation between the techniques. These data highlight the issues and perils of making comparisons across techniques in the published literature, as the ultimate results are dependent on the conditions and equipment used. However, similar trends in data across groups indicate that even though these techniques vary in experimental conditions leading to differences in the measured parameters, each is a valid technique to assess different components of lymphatic function. Using a single lymphatic measurement technique to compare control and experimental situations is important.

One experimental condition effect that is difficult to assess with this procedure is how the time after injection alters lymphatic parameters. Due to the measurement of contraction before and after skin removal, this was not possible here. Others have shown that frequency is affected by time after injection; initially there is a large increase in frequency that later normalizes (23). Similar experiments have been completed in ex vivo preparations (19). In an effort to minimize this dramatic effect, we waited an hour after injection before imaging with NIRF, but it is difficult to remove the effect of time completely.

In addition, there have been several studies suggesting dramatic effects of contrast agents, particularly ICG, on lymphatic function $(13,22,23)$. To remove these effects, the same contrast agent was used for all 


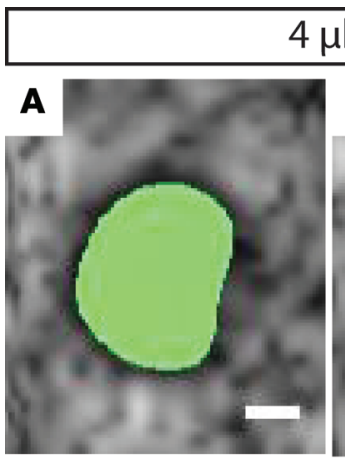

\section{$4 \mu \mathrm{l}$}
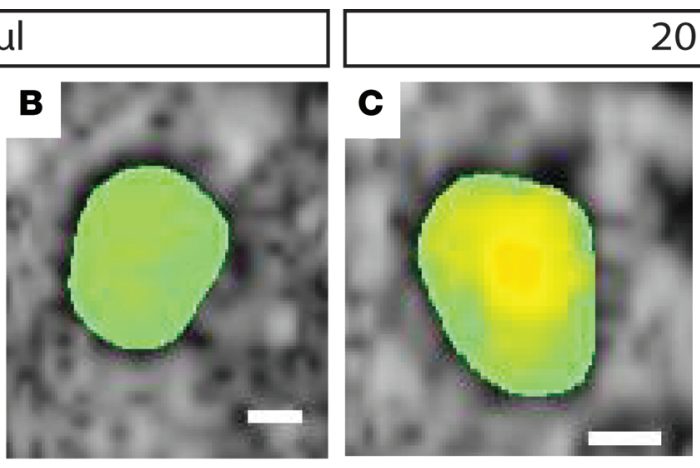

$20 \mu \mathrm{l}$
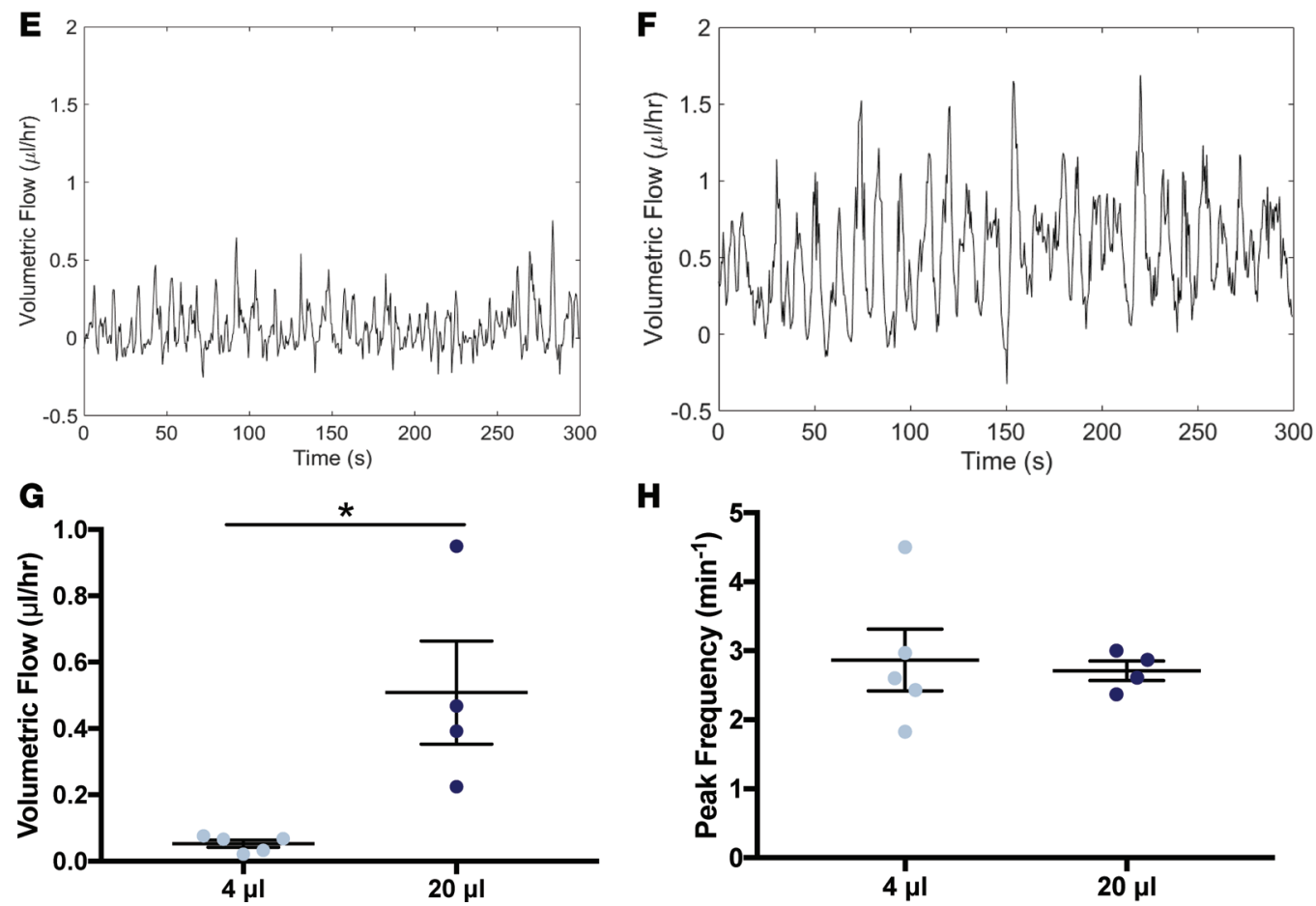

Figure 5. Larger injection volume increases volumetric flow in lymphatic vessels. (A-D) Mice injected with $4 \mu \mathrm{l}$ or $20 \mu \mathrm{l}$ of a $50: 50$ mixture of FITC-dextran and indocyanine green (ICC) in the footpad 1 hour prior to Doppler optical coherence tomography (DOCT) imaging. Representative images of mice injected with (A and B) $4 \mu \mathrm{l}$ or (C and D) $20 \mu \mathrm{l}$ during low volumetric flow (A and $\mathbf{C}$ ) versus a spike in flow (B and D). Mean volumetric flow over the cross section for (E) $4 \mu \mathrm{l}$ and (F) $20 \mu \mathrm{l}$ averaged over 300 seconds to give the average volumetric flow $\left(\mathbf{G}, n=4-5\right.$, ${ }^{*} P<0.05$ by Mann-Whitney test). Note the larger volumetric flow in mice injected with $20 \mu \mathrm{l}$, even between lymphatic contractions (C, quantified in $\mathbf{F}$ and $\mathbf{G}$ ). However, no difference was found in the frequency of peaks of volumetric flow $\mathbf{( H )}$. Scale bars: $20 \mu \mathrm{m}$. Data presented as the mean $\pm \mathrm{SEM}$.

experiments, but future work is warranted to determine if different contrast agent concentrations would have affected the conclusions of this study. Further, removing skin showed a reduction in lymphatic contraction measured by NIRF (Figure 2D). While it is unclear why there was such a dramatic decrease, it is hypothesized that this is due to the superfusion of vessels with saline that increases shear or from a reduction in confinement/extramural compression from removing the skin.

Lastly, it was found that even though the contraction frequency and NIR frequency were lower following a $20-\mu 1$ injection versus a $4-\mu 1$ injection, there was a significant 10 -fold greater volumetric flow in mice injected with $20 \mu 1$ versus $4 \mu 1$ (Figure 5). Previous measurements after no injection found a similar frequency (3.0 $\pm 0.3 / \mathrm{min})$ to that following a $4-\mu 1$ and $20-\mu 1$ injection (17). Similarly, volumetric flow with no injection was found to be approximately $0.03 \pm 0.01 \mu \mathrm{l} / \mathrm{hr}$, which is comparable to the $4-\mu 1$ measurement (8).

Lymphatic contraction reduction in the presence of increased flow suggests there is flow-mediated dilation and contraction inhibition due to the presence of a favorable upstream pressure gradient, which has been reported via ex vivo analysis of lymphatic vessels subjected to step-wise increasing pressures $(18,20,24$ 26). Similar results of increased lymph flow have been reported with intravital imaging of rats experiencing 
edemagenic stress (30). Interesting, there was no difference in the frequency of the peaks in volumetric flow (Figure $5 \mathrm{H}$ ), suggesting that these peaks in volumetric flows are at least partially independent of lymphatic contraction and could be dependent on valve dynamics (31). These data show that restricting the analysis to only lymphatic contraction parameters is not sufficient to get a full understanding of lymphatic function. Several disease states show decreased lymphatic contraction but increased collecting lymphatic vessel diameter $(2,16,32)$. Thus, it is possible that there is increased flow in these situations. Future work is needed to characterize lymph flow in these disease processes.

\section{Methods}

Mice. In all experiments, 8- to 10-week-old C57BL/6 female mice were used. Before imaging, mice were anesthetized using a ketamine/xylazine mixture at $100 \mathrm{mg} / 10 \mathrm{mg}$ per $\mathrm{kg}$ body weight. The footpad was then injected with 4, 10, or $20 \mu \mathrm{l}$ of a 50:50 mixture of $0.5 \mathrm{mg} / \mathrm{ml}$ ICG (Pulsion Medical Systems) and 2\% 2,000-kDa-dextran-conjugated fluorescein isothiocyanate (FITC-dextran) (Sigma-Aldrich) 1 hour prior to imaging. Imaging sessions were completed within an additional 1 hour. Mice were euthanized at the end of the imaging sessions. For Figures 2 and 3, mice first underwent NIR imaging with skin intact; then, the skin was removed and the mice were imaged again with NIRF. Subsequently, mice underwent direct fluorescence imaging of lymphatic contraction.

NIRF imaging. Mice had hair removed by a depilatory cream and were kept at $37^{\circ} \mathrm{C}$ by being placed on a heating pad. They were then imaged with a $\times 6.5$ Zoom lens (Navitar), and a Prosilica GT2750 camera (Allied Vision Technology) with an ICG-B emission filter (832/37, Semrock). MATLAB (MathWorks) was used to control the camera settings and record images. A custom-built ring light source was used to excite the ICG using $760 \mathrm{~nm}$ high-power laser-emitting diodes (Marubeni) filtered through a 775/50 bandpass filter (Chroma). After imaging noninvasively, the skin on the dorsal side of the mouse hindlimb was surgically removed and tissue was kept hydrated using physiological saline ( $0.9 \%$ sodium chloride, Baxter) for further imaging. ImageJ (NIH) was used to analyze the mean signal intensity of 3 ROIs along the lymphatic vessel. All NIR imaging was done prior to direct measurement of lymphatic contraction using fluorescence microscopy.

Direct measurement of lymphatic contraction using fluorescence microscopy. Intravital lymphatic fluorescence imaging was performed as previously described $(9,16)$. Briefly, after hair removal via a depilatory cream, the skin on the dorsal side of the mouse hindlimb was surgically removed. Exposed tissue was kept hydrated using physiological saline and kept at $37^{\circ} \mathrm{C}$ by a custom-built water bath. Mice were then placed either sitting upright or in a supine position, and lymphatic vessels were imaged using an inverted Olympus microscope. Several points along the lymphatic vessels were imaged and subsequently analyzed using MATLAB to track the vessel wall movement as described previously (9). Multiple location analyses were averaged to give a single value for each mouse.

DOCT. Mice were prepared as described for fluorescence imaging. After surgery, mice were positioned on a stage tilted $15^{\circ}$ with the hindlimb tilted downward, secured with surgical tape. The angle provides a gravity-induced pressure as would be present in the hindlimb of an ambulating mouse and ensures the vessel is at an angle to the imaging axis, which is necessary for Doppler analysis. Mice were imaged on an OCT system as previously described (17). Briefly, the system uses a swept-wavelength laser source centered at 1,300 $\mathrm{nm}$. The lymphatic vessel was identified from real-time OCT B-scan images, then cross sections from one vessel were continuously acquired for 300 seconds. This was repeated 3 times and averaged for each mouse. Processing was performed in MATLAB as previously described $(17,33)$. Briefly, the temporal power spectral density was calculated over the B-scans for each pixel and was then fitted by 2 constrained circular Gaussians, the first one centered at $0 \mathrm{~Hz}$ representing tissue and the second Gaussian shifted from $0 \mathrm{~Hz}$ estimating the Doppler frequency shift by its center. The frequency was converted to the flow velocity using the vessel Doppler angle. The segmented vessel area was used to calculate the volumetric flow. For display purposes in the videos, a median filter over 3 temporal frames was applied for each spatial pixel in order to remove localized noise or points of velocity noise or artifact.

Statistics. Normality was tested using a Shapiro-Wilk normality test and significance was determined by ANOVA (Fisher's LSD post-hoc test) or 2-tailed $t$ test for normally distributed data. $P$ values less than 0.05 were considered significant. All analysis was performed in Prism (GraphPad).

Study approval. The protocol for the animal experiments was reviewed and approved by the Institutional Animal Care and Use Committee of the Massachusetts General Hospital. The procedures were performed in accordance with the approved guidelines. 


\section{Author contributions}

EMB, CB, and EFJM were responsible for conducting experiments, acquiring data, and analyzing data. TAR designed and constructed the NIR light source. EMB, CB, LLM, BJV, and TPP designed experiments and interpreted data. All authors contributed to writing and reviewing the manuscript.

\section{Acknowledgments}

This work was supported by NIH grants DP2 OD008780 and R01 HL128168. CB was supported by a Swiss National Science Foundation Early Postdoctoral Mobility fellowship (P2SKP2_158640). EMB was supported by the LE\&RN/FDRS Lipedema Postdoctoral Fellowship.

Address correspondence to: Timothy P. Padera, Massachusetts General Hospital, Radiation Oncology-Cox-737, 100 Blossom Street, Boston, Massachusetts 02114, USA. Phone: 617.643.6920; Email: tpadera@steele.mgh.harvard.edu.

1. Iliff JJ, et al. A paravascular pathway facilitates CSF flow through the brain parenchyma and the clearance of interstitial solutes, including amyloid $\beta$. Sci Transl Med. 2012;4(147):147ra111.

2. Li J, et al. Efficacy of B cell depletion therapy for murine joint arthritis flare is associated with increased lymphatic flow. Arthritis Rheum. 2013;65(1):130-138.

3. Aspelund A, et al. The Schlemm's canal is a VEGF-C/VEGFR-3-responsive lymphatic-like vessel. J Clin Invest. 2014;124(9):3975-3986.

4. Wiig H, et al. Immune cells control skin lymphatic electrolyte homeostasis and blood pressure. J Clin Invest. 2013;123(7):2803-2815.

5. Klotz L, et al. Cardiac lymphatics are heterogeneous in origin and respond to injury. Nature. 2015;522(7554):62-67.

6. Hoshida $\mathrm{T}$, et al. Imaging steps of lymphatic metastasis reveals that vascular endothelial growth factor-C increases metastasis by increasing delivery of cancer cells to lymph nodes: therapeutic implications. Cancer Res. 2006;66(16):8065-8075

7. Munn LL, Padera TP. Imaging the lymphatic system. Microvasc Res. 2014;96:55-63.

8. Blatter C, et al. In vivo label-free measurement of lymph flow velocity and volumetric flow rates using Doppler optical coherence tomography. Sci Rep. 2016;6:29035.

9. Liao S, Jones D, Cheng G, Padera TP. Method for the quantitative measurement of collecting lymphatic vessel contraction in mice. J Biol Methods. 2014;1(2):e6.

10. Robinson HA, Kwon SK, Hall MA, Rasmussen JC, Aldrich MB, Sevick-Muraca EM. Non-invasive optical imaging of the lymphatic vasculature of a mouse. J Vis Exp. 2013;(73):e4326.

11. Rahimi H, et al. Lymphatic imaging to assess rheumatoid flare: mechanistic insights and biomarker potential. Arthritis Res Ther. 2016;18:194.

12. Sevick-Muraca EM, Kwon S, Rasmussen JC. Emerging lymphatic imaging technologies for mouse and man. J Clin Invest. 2014;124(3):905-914.

13. Proulx ST, et al. Use of a PEG-conjugated bright near-infrared dye for functional imaging of rerouting of tumor lymphatic drainage after sentinel lymph node metastasis. Biomaterials. 2013;34(21):5128-5137.

14. Chong $C$, et al. In vivo visualization and quantification of collecting lymphatic vessel contractility using near-infrared imaging. Sci Rep. 2016;6:22930.

15. Weiler M, Kassis T, Dixon JB. Sensitivity analysis of near-infrared functional lymphatic imaging. J Biomed Opt. 2012;17(6):066019.

16. Liao S, et al. Impaired lymphatic contraction associated with immunosuppression. Proc Natl Acad Sci USA. 2011;108(46):18784-18789.

17. Blatter C, et al. In vivo label-free measurement of lymph flow velocity and volumetric flow rates using Doppler optical coherence tomography. Sci Rep. 2016;6:29035.

18. Gashev AA, Davis MJ, Delp MD, Zawieja DC. Regional variations of contractile activity in isolated rat lymphatics. Microcirculation. 2004;11(6):477-492.

19. Scallan JP, Wolpers JH, Muthuchamy M, Zawieja DC, Gashev AA, Davis MJ. Independent and interactive effects of preload and afterload on the pump function of the isolated lymphangion. Am J Physiol Heart Circ Physiol. 2012;303(7):H809-H824.

20. Koller A, Mizuno R, Kaley G. Flow reduces the amplitude and increases the frequency of lymphatic vasomotion: role of endothelial prostanoids. Am J Physiol. 1999;277(6 Pt 2):R1683-R1689.

21. Davis MJ, Davis AM, Lane MM, Ku CW, Gashev AA. Rate-sensitive contractile responses of lymphatic vessels to circumferential stretch. J Physiol (Lond). 2009;587(1):165-182.

22. Gashev AA, Nagai T, Bridenbaugh EA. Indocyanine green and lymphatic imaging: current problems. Lymphat Res Biol. 2010;8(2):127-130.

23. Weiler M, Dixon JB. Differential transport function of lymphatic vessels in the rat tail model and the long-term effects of Indocyanine Green as assessed with near-infrared imaging. Front Physiol. 2013;4:215.

24. Scallan JP, Wolpers JH, Muthuchamy M, Zawieja DC, Gashev AA, Davis MJ. Independent and interactive effects of preload and afterload on the pump function of the isolated lymphangion. Am J Physiol Heart Circ Physiol. 2012;303(7):H809-H824

25. Kunert C, Baish JW, Liao S, Padera TP, Munn LL. Mechanobiological oscillators control lymph flow. Proc Natl Acad Sci USA. 2015;112(35):10938-10943.

26. Baish JW, Kunert C, Padera TP, Munn LL. Synchronization and random triggering of lymphatic vessel contractions. PLoS Comput Biol. 2016;12(12):e1005231. 
27. Benoit JN, Zawieja DC, Goodman AH, Granger HJ. Characterization of intact mesenteric lymphatic pump and its responsiveness to acute edemagenic stress. Am J Physiol. 1989;257(6 Pt 2):H2059-H2069.

28. Kornuta JA, Nepiyushchikh Z, Gasheva OY, Mukherjee A, Zawieja DC, Dixon JB. Effects of dynamic shear and transmural pressure on wall shear stress sensitivity in collecting lymphatic vessels. Am J Physiol Regul Integr Comp Physiol. 2015;309(9):R1122-R1134.

29. Scallan JP, Wolpers JH, Davis MJ. Constriction of isolated collecting lymphatic vessels in response to acute increases in downstream pressure. J Physiol (Lond). 2013;591(2):443-459.

30. Rahbar E, Akl T, Coté GL, Moore JE, Zawieja DC. Lymph transport in rat mesenteric lymphatics experiencing edemagenic stress. Microcirculation. 2014;21(5):359-367.

31. Blatter C, Meijer EFJ, Padera TP, Vakoc BJ. Simultaneous measurements of lymphatic vessel contraction, flow valve dynamics in multiple lymphangions using optical coherence tomography [published online ahead of print July 12, 2017]. J Biophotonics. https://doi.org/10.1002/jbio.201700017.

32. Blum KS, et al. Chronic high-fat diet impairs collecting lymphatic vessel function in mice. PLoS One. $2014 ; 9(4):$ e94713.

33. Bouma BE, Yun SH, Vakoc BJ, Suter MJ, Tearney GJ. Fourier-domain optical coherence tomography: recent advances toward clinical utility. Curr Opin Biotechnol. 2009;20(1):111-118. 